Revue européenne des sciences sociales

European Journal of Social Sciences

XLV-136 | 2007

Démocratie délibérative, démocratie débattante, démocratie participative

\title{
Voter et délibérer
}

Pasquale Pasquino

\section{(2) OpenEdition}

Journals

Édition électronique

URL : http://journals.openedition.org/ress/84

DOI : $10.4000 /$ ress. 84

ISSN : 1663-4446

Éditeur

Librairie Droz

Édition imprimée

Date de publication : 1 février 2007

Pagination : $35-45$

ISBN : 978-2-600-01114-3

ISSN : 0048-8046

Référence électronique

Pasquale Pasquino, "Voter et délibérer », Revue européenne des sciences sociales [En ligne],

XLV-136 | 2007, mis en ligne le 01 février 2010, consulté le 01 mai 2019. URL : http://

journals.openedition.org/ress/84; DOI : 10.4000/ress.84 


\section{Pasquale PASQUINO}

\section{VOTER ET DÉLIBÉRER}

Dans quelques travaux récents consacrés, avec John Ferejohn, au contrôle de constitutionnalité comparé ${ }^{1}$, nous avons proposé de caractériser les cours constitutionnelles $^{2}$ en tant qu'organes délibératifs, en distinguant, d'ailleurs, les cours à délibération «interne» (notamment la Cour Constitutionnelle italienne ou le Conseil Constitutionnel français) de celle à délibération «externe» (par exemple la Court Suprême des Etats-Unis). Or, dans aucun de ces textes nous n'avons proposé une définition analytique du concept de délibération, ce que je voudrais essayer de faire pour ma part ici, à l'aide d'une dichotomie heuristique.

Jon Elster, dans un article maintenant classique ${ }^{3}$, a opposé «arguing and bargaining», qu'il qualifie de speech acts; je vais opposer, en revanche et de manière idéal-typique, deux modalités de prise de décision collective. Par décision collective, il faut comprendre ici toute décision prise par un groupe ou un collège, laquelle lie tous les membres du groupe, et, s'il s'agit d'un groupe de décideurs représentatifs ${ }^{4} \mathrm{~d}^{\prime} u n e$ communauté plus large, aussi tous les membres représentés. Les modalités de décision collective que je vais considérer sont:

a) le vote, et

b) la délibération.

Par vote, j'entends ici une décision ${ }^{5}$ - secrète ou publique - prise en dénombrant les volontés individuelles d'un nombre $\mathbf{x}$ d'individus considérés égaux par

1 «Constitutional Courts as Deliberative Institutions: Towards an Institutional Theory of Constitutional Justice», in: W. Sadurski (ed.), Constituional Justice, East and West, The Hague, etc., Kluver Law International, 2002, pp. 21-36 and «Constitutional Adjudication: Lessons from Europe», in Texas Law Review, (2004), volume 82, issue 7, pp. 1671-1704.

2 Ici, et toujours dans cet article, au sens large de l'expression, qui inclut aussi bien les cours spécialisées (comme la Corte Costituzionale italienne et le Bundesverfassungsgericht) que les Cours Suprêmes avec compétence de contrôle de constitutionnalité et le Conseil constitutionnel - qui n'est pas à proprement parler une juridiction.

3 «Argumenter et négocier dans deux Assemblées constituantes», in Revue Française de Science Politique, vol. 44, $\mathrm{n}^{\circ}$ 2, avril 1994, pp. 187-256

4 Le mot «représentant»a ici son sens le plus général de quelqu'un qui est autorisé, par une norme quelconque, à prendre des décisions pour d'autres que lui-même, indépendamment, par exemple, de toute responsabilité vis-à-vis de ces autres.

5 Si l'on exclut que la décision soit prise à la «pluralité» des suffrages, donc par la minorité la plus importante, il faut que les décideurs soient en présence d'un choix binaire (entre ' $a$ ' et 'b' ou 'a' et 'non-a'), ce qui demande des mécanismes capables de réduire à deux les alternatives en présence avec les paradoxes qu'on connaît. 
la procédure. Par l'adjectif «égaux», je fais référence au fait que les décideurs ont le même poids dans le résultat du vote - une condition essentielle de la règle de majorité.

La forme pure de ce mécanisme de prise de décision collective était utilisée par les dikasteria, les tribunaux populaires de la démocratie athénienne du IV siècle av. J.-C. Dans cet organe fondamental du système institutionnel de l'époque de Démosthène, le vote était secret, égal et ne suivait pas une discussion entre les dikastai, les jurés populaires. La discussion était en réalité interdite, et ceci pour des raisons que je vais évoquer plus loin, et qui sont liées, entre autre, à la protection du secret. Les jurés se limitaient, donc, à voter, à la suite du «contradictoire» entre les parties du procès. Par exemple, dans le cas d'une graphé paranómon ${ }^{6}$, ils devaient dire si "oui" ou "non" la décision de l'assemblée - pséphisma-, attaquée en justice par le citoyen ayant porté plainte, était ou n'était pas contraire aux règles politiques et constitutionnelles du régime populaire athénien ${ }^{7}$.

En outre, et c'est peut être le caractère essentiel de ce type idéal, le votant / l'électeur est souverain au sens du souverain de l'époque baroque qui peut dire de ses décisions: Sic volo, sic jubeo, stat pro ratione voluntas, je décide comme je veux et ma volonté souveraine vaut en tant qu'argument (ou raison). Ou aussi, dans la version égalitaire: mon argument est «mon poids égal dans la décision», mon être un «fragment égal» de la souveraineté ${ }^{8}$.

Par délibération ${ }^{9}$, j'entends, par une définition stipulative, une modalité de décision collective qui tend à exclure le vote, qui ne considère pas celui-ci comme le mécanisme essentiel de la prise de décision. Pour illustrer ma thèse, je vais faire référence à deux cas de prises de décision réelles que je connais un peu de près: les «sentenze» [arrêts] de la Cour Constitutionnelle italienne, donc des décisions qui,

6 Cette expression désigne l'accusation portée par un citoyen devant le tribunal à l'encontre d'un autre citoyen qui avait proposé lors d'une réunion de l'assemblée (l'ekklesia) le texte d'une décision approuvée par l'assemblée mais que le citoyen plaignant considère contraire aux nomoi (les lois et le régime démocratique) d'Athènes.

7 Voir: H. J. Wolff, Normenkontrolle und Gesetzesbegriff in der attischen Demokratie. Untersuchungen zur graphé paranomon, Sitzungsberichte der Heidelberger Akademie der Wissenschaften, Philos.-hist. Klasse, 1970, 2. Abh.; et M. H. Hansen, The Sovereignty of the People's Court in Athens in the Fourth Century B.C. and the Public Action against Unconstitutional Proposals, Odense, 1974.

8 Philippe Urfalino, que je tiens à remercier, me fait remarquer à raison que le vote en tant que mécanisme de décision collective est indépendant du principe de majorité. On peut, en fait, imposer une règle autre que celle de la majorité absolue, par exemple une majorité qualifiée ou même l'unanimité. Je crois néanmoins que le vote à majorité simple ou absolue possède une propriété spécifique essentielle à la typologie proposée ici et est lié à une option normative importante, sur laquelle je vais revenir dans le texte. Il est le seul compatible avec l'idée d'égalité stricte (le poids égal) entre les acteurs de la décision. Ce qui mériterait une analyse beaucoup plus développée, dont je dois faire abstraction ici. Il suffira de dire que la majorité qualifiée introduit un biais en faveur du statu quo qui casse le principe d'égalité par lequel je caractérise mon mécanisme de prise de décision. En fait, si la règle demande une majorité de $60 \%, 41 \%$ pèsent plus que $59 \%$ pour assurer le maintien du statu quo. Dans le cas du vote unanime, toutes les voix pèsent également, mais seulement dans le sens que chaque voix peut également empêcher toute décision collective!

9 Dans le langage commun, le terme est utilisé dans deux acceptions: l'Oxford English Dictionary, par exemple distingue: «careful consideration, weighing up with a view to decision; debate», de la signification obsolète de «resolution, determination». La définition qu'on propose ici lie entre eux les deux sens du terme. 
on le verra, sont le résultat d'un type particulier de procédure entre les membres qui composent le collège, d'un côté; et, de l'autre, les débats qui ont abouti au nouveau Traité constitutionnel préparé par «Convention pour l'avenir de l'Europe».

Remarquons, pour commencer, que le débat, qui est en amont de la décision et qui la produit, est dans le cas d'une cour constitutionnelle, secret - on ne sait pas, en principe $^{10}$, ce qui se passe lors de la discussion entre les juges constitutionnels -, on connaît simplement le résultat de la discussion: la motivation de la sentence, qui est signée par tous les membres de la cour et qui exprime le «droit» plutôt que l'opinion d'une majorité de juges ${ }^{11}$. Deuxièmement, les juges sont formellement égaux (à l'exception du président, dans certains cas), mais dès que le débat s'engage, le principe ou la règle d'égalité se heurte à des différences factuelles incontournables. Celles-ci apparaissent du fait même de la pratique de l'argumentation / délibération (que j'oppose ici au vote).

Ces différences découlent de plusieurs sources:

1. l'autorité personnelle de l'orateur (son caractère ${ }^{12}$, sa réputation d'impartialité, etc.);

2. la compétence technique (un spécialiste de procédure criminelle ne peut pas facilement - ceteris paribus - s'opposer à son collègue professeur de droit administratif sur des questions de stricte compétence du dernier; un universitaire aura du mal à l'emporter dans une discussion avec son collègue qui vient de la Cour de Cassation à propos d'une question de constitutionnalité relative au fonctionnement, par exemple, des cours d'appel, etc.);

3. l'intelligence et la puissance argumentative vis-à-vis des autres partenaires de la discussion (on sait, par exemple, qu'il était difficile de s'opposer au sein du Conseil Constitutionnel à Robert Badinter (à cause de 1,3, et parfois 2!) ${ }^{13}$. Il faut ajouter, en outre,

4. comme Jon Elster me le fait remarquer, que la succession dans laquelle les juges interviennent dans la discussion joue aussi un rôle dans la prise de décision;

${ }^{10}$ On sait tout de même quelques choses par ce qu'en disent les juges constitutionnels; voir par exemple de Noëlle Lenoir, «Le Métier de juge constitutionnel», Le Débat, n 114, mars-avril 2001, p. 178 sqq.

${ }^{11}$ Il est évident que je parle d'un collège de juges typique de la tradition juridique française, qui ne connaît pas d'opinion dissidente et où la cour parle toujours avec une seule voix; ce qui n'est pas du tout le cas des cours anglo-américaines, qui s'inspirent de l'ancienne tradition des décisions collégiales seriatim (où chaque membre du collège judiciaire prépare et présente sa décision individuellement et en succession)!

12 «On persuade par le caractère, quand le discours est de nature à rendre l'orateur digne de foi, car les honnêtes gens nous inspirent confiance plus grande et plus prompte sur toutes les questions en général... c'est le caractère qui, peut-on dire, constitue presque la plus efficace des preuves», Aristote, Rhétorique, 1356a (trad. de M. Dufour, Gallimard, 1998, pp. 22-23). Ce point est repris par Ph. Urfalino dans son texte: «La délibération et la dimension normative de la décision collective» paru in: La juridicisation du politique, sous la direction de J. Comaille et alii, L.G.D.J, 1999, pp. 165-193.

13 J'admets qu'il n'est pas toujours aisé de distinguer 1 et 3 . 
5. et que dans ce type de prise de décision - à la différence de ce qui se passe dans le vote - une fonction très importante est jouée par le président de l'assemblée ou du collège délibérant. Car c'est à lui qu'échoit la tâche de tirer du débat la conclusion, un point qu'il faudrait considérer en détail - on peut penser, par exemple, au rôle décisif du président V. Giscard d'Estaing dans le travail qui a conduit à la rédaction du traité constitutionnel pour l'Union Européenne.

Je reviens brièvement sur le caractère secret de la prise de décision, car on le retrouve dans mes deux exemples de décision collective ${ }^{14}$. Il faut remarquer que dans le premier cas, celui du vote, le secret protège celui qui vote et lui permet de faire l'économie d'un argument (donné aux autres - chacun peut se donner des arguments à lui-même ${ }^{15}$, mais ceci ne nous concerne pas ici, dans le contexte des décisions collectives). Dans le cas de la délibération, le secret protège l'organe de la décision non le membre individuel de celui-ci, qui au contraire est obligé de présenter un argument à ses partenaires, de même que l'organe est obligé de présenter au public les raisons de sa décision (la motivation) - ce qui n'est évidemment pas le cas du vote (qui peut être décrit comme une simple agrégation, mécanique et sans raisons, de préférences individuelles).

C'est en tenant compte de ce qu'on vient de dire, que je suggère d'appeler mon deuxième type idéal un mécanisme délibératif de prise de décision, au sens où chaque membre de l'organe est obligé de présenter un argument - une bonne raison de choisir ' $\mathrm{x}$ ', une raison, plus précisément, qui doit pouvoir être acceptée par les autres et qui ne peut donc pas être présentée comme une préférence personnelle ou d'une partie de l'organe - même si elle peut être présentée, dans certains cas et pas dans la motivation publique, comme une préférence de l'organe dans son ensemble ${ }^{16}$ - on peut aussi penser à l' «ambition» dont parle James Madison dans le cadre de sa doctrine de la balance des pouvoirs. Mécanisme délibératif aussi au sens où la décision collective elle-même prend la forme d'un «argument», plutôt que de la décision typiquement démocratique du «oui» ou «non» ${ }^{17}$.

Dans ce type idéal, le résultat de la décision collective ne doit pas être le simple produit d'un vote, qui opposerait la majorité à la minorité en forçant celleci à accepter purement et simplement la volonté de la première (quoiqu'il puisse arriver exceptionnellement de voter pour éviter d'éterniser le débat, ou pour avoir une photographie de la discussion à un moment donné).

${ }^{14}$ Le vote secret caractérise souvent les assemblées d'électeurs et les assemblées populaires qui doivent prendre des décisions (référendum); dans le cas des assemblées représentatives, le caractère public du vote est essentiel pour garantir la responsabilité des élus devant le suffrage. Le vote secret dans ce dernier type d'assemblées a été justifié, néanmoins, dans des contextes différents en tant que garantie pour les élus vis-à-vis du monarque ou de la direction du parti qui pourrait imposer à ses membres, sous menace de rétorsions, de voter contre leur conscience - encore une question que je puis qu'évoquer ici.

${ }^{15}$ Voir: R. Boudon, L'art de se persuader, Paris, Fayard, 1990.

16 J'ai à l'esprit ici à un argument du type: «Il faut choisir ' $\mathrm{x}$ ', car c'est la décision plus favorable à la réputation d'impartialité de la Cour».

${ }^{17}$ Sur ce dernier point voir de Erich Kaufmann, Zur Problematik des Volkswillens, Berlin, 1931. 
Ce que j'appelle ici “argument”, une pièce centrale du mécanisme de la délibération, a sans doute un «air de famille» avec ce que John Rawls dans son Libéralisme Politique définit comme "public reason» (sur ce même concept Rawls est revenu dans sa postface à The law of peoples) ${ }^{18}$. Je ne vais pourtant pas m'engager ici dans un commentaire du texte Rawlsien. Je préfère revenir à ma dichotomie pour en développer quelques aspects conceptuels.

On pourrait objecter, concernant mon exemple de délibération, tout d'abord, que la sentence d'une cour constitutionnelle consiste, exactement comme pour les tribunaux populaires athéniens, à trancher par un «oui» ou «non» la question soulevée devant elle à propos de la constitutionnalité d'une loi. Cette vision est trop simpliste, et, essentiellement, fausse. Les sentences se présentent (en Italie, mais dans pratiquement tous les autres cas que je connais) comme une interprétation constitutionnelle, comme une réécriture de la loi, qui tranche très rarement par un simple "oui" ou "non" la question posée de la constitutionnalité de celle-ci (et aux Etats-Unis, comme un «précédent» qui redéfinit les rapports entre la Cour Suprême et les autres branches du gouvernement - que l'on pense aux "opinions" de l'été 2004 concernant les prisonniers de la baie de Guantanamo - donc, comme une redéfinition des rapports entre organes constitutionnels) ${ }^{19}$.

On peut objecter, par ailleurs, que la décision - «oui» ou «non»-d'un référendum populaire se produit aujourd'hui (comme celle de l'ekklesia, l'assemblée populaire athénienne, où le vote était d'ailleurs ouvert, à main levée) à la fin d'un débat public. Certes. Mais la décision se fait, dans ce cas de figure, en additionnant les voix, non les arguments. On peut certes croire que les arguments échangés avant le vote déterminent les décisions individuelles, et infléchissent ou même produisent les préférences exprimées par le vote. Peut-être, mais comment le savoir, étant donné que le vote est secret? ${ }^{20}$

En revanche, dans le cas de la décision judiciaire d'un collège de juges, surtout si comme en France ou en Italie il n'y a pas de dissenting opinion, la décision doit être le résultat du débat qui a opposé arguments à arguments, et qui doit finir par les intégrer. Aucun choix ne peut être fait sans "giving a reason», et même, je pense, «a public reason», une raison qui ne peut pas se réduire à l'expression de l'intérêt ou même à l'idéologie politique de celui qui parle. D'où le paradoxe suivant: dans le secret de «la camera di consiglio», de la délibération, les juges sont obligés de donner des raisons «publiques», tandis qu'au bout d'un débat public, les électeurs, dans le secret de l'urne, peuvent faire valoir légitimement, et malgré ce qu'en dit Rousseau, leurs intérêts ou préférences privés!

18 «The Idea of Public Reason Revisited», pp. 129-180, Harvard University Press, 2001.

19 «Justice Anthony M. Kennedy said he discussed the power of the United States Supreme Court by citing clashes between the courts and the president in which the presidency lost but had to accept the rule of law.» The New York Times, 6 mai 2004.

${ }^{20}$ Un petit peu par les sondages, mais on est là sur un terrain incertain. D'un côté, il y a toujours ceux - certes sans doute moins nombreux - qui n'écoutent pas, et qui votent toujours de la même manière. De l'autre, ceux - et ils sont de plus en plus nombreux - qui se déterminent à la dernière minute et on ne sait pas trop pourquoi! Renato Mannheimer me fait observer que cette décision de dernière minute porte aussi bien sur le fait de voter ou pas que sur le choix du parti ou du candidat. 
Il n'est pas étonnant, soit dit en passant, que les universitaires aiment la «démocratie délibérative», car elle fait rentrer par la fenêtre ce que le suffrage universel avait chassé par la porte: le refus de l'égalité stricte dans la prise de décision et la souveraineté de la sanior pars plutôt que de la major pars ${ }^{21}$. La Démocratie Délibérative serait donc une variante de ce que souhaitait J. S. Mill: un vote plus ou moins "pesant" pour chacun, sur la base de la qualité de l'individu délibérant / décideur. Ici on défend, en revanche, l'idée que vote et délibération, en tant que mécanismes de prise de décision collective, appartiennent tous les deux aux règles d'un bon gouvernement dans des contextes institutionnels différents qu'une théorie plus développée devrait s'efforcer de préciser.

On peut objecter, enfin, qu'un argument ne fait jamais rien d'autre que cacher une passion ou un intérêt. Mais si les autres locuteurs / décideurs ne sont pas benêts, il faut que celui qui cache son intérêt (ou la passion qui le motive) aille un peu plus loin, qu'il ne se limite pas à masquer celui-ci par des simples astuces rhétoriques. Qu'il en présente, en somme, une version moins vulgaire ${ }^{22}$, qu'il concède quelque chose sur la substance de sa position à ses interlocuteurs! De sorte que pour paraître moins vulgaire, il se doit de l'être moins, en réalité! Sur ce point, je ne puis qu'être d'accord avec Jon Elster lorsqu'il parle avec La Rochefoucauld de la «valeur civilisatrice de l'hypocrisie»! !3

La décision fondée sur le vote suppose que toutes les options soient égales (même si l'on n'est pas obligé de penser qu'elles soient également bonnes). La décision fondée sur un échange d'arguments suppose, en principe, qu'il y a des arguments meilleurs, qui doivent (ou devraient) l'emporter, par effet de la persuasion.

On peut alors se demander comment on reconnaît qu'un argument est meilleur qu'un autre. On peut, certes, voter sur les arguments. On reviendrait, dans ce cas, au premier terme de ma dichotomie, la décision par le vote. Mais on peut aussi décider de ne pas voter, comme dans le cas de ma délibération, et accepter une alternative au vote qu'on pourrait appeler aussi «décision par consensus» ${ }^{24}$.

Prenons, pour éclaircir cet aspect essentiel du deuxième type de décision collective, le cas, dont on a beaucoup discuté récemment, de la ratification d'un traité international. Dans ce cas, on ne vote pas, et ceci pour une raison assez simple: soit on est tous d'accord - et, donc, ce n'est pas la peine de voter -, soit il n'y a pas l'unanimité et ce n'est pas la peine de voter non plus! J'ai, pour ma part, autre chose à l'esprit lorsque je parle de «décision par consensus». Je pense à une situation dans laquelle, puisqu'il est exclu de décider par le simple recours au vote à

${ }^{21}$ On trouve quelques remarques intéressantes sur ce point dans l'article de Lynne Sanders, «Against Deliberation», Political Theory, 25 (1997), pp. 347-375.

${ }^{22}$ Au sens de self-interested.

${ }^{23}$ Maxime 218, éd. J. Lafond, Gallimard, 1976: «L'hypocrisie est un hommage que le vice paye à la vertu» - Lafont fait remarquer (p. 291) que l'idée est déjà chez Du Moulin, Traité de la paix de l'âme, 1660.

${ }^{24}$ Un sujet sur lequel travaille de manière très intéressante, et d'un point de vue sociologique et anthropologique, Philippe Urfalino. 
majorité ${ }^{25}$, il faut trouver un compromis entre tous les arguments présentés. Ce qui est plus facile s'il s'agit d'un groupe restreint de locuteurs / décideurs (les quinze juges de la Cour Constitutionnelle italienne ou les neuf membres du Conseil Constitutionnel français); beaucoup plus difficile si le nombre des décideurs est important ${ }^{26}$. Dans ce cas, on peut procéder comme on l'a fait lors des travaux de la Convention pour l'avenir de l'Europe. Ici, les propositions venant des membres de la Convention étaient étudiées par le Secrétariat, discutées, ensuite, par un organe restreint, le Présidium, et enfin, étaient l'objet d'une nouvelle discussion dans l'assemblée plénière, sans qu'il y ait jamais de vote ni dans le Présidium ${ }^{27}$, ni dans les plénières ${ }^{28}$.

Avant de considérer cette expérience, je voudrais faire encore une remarque d'ordre général. On pourrait distinguer entre «compromis» et «consensus», en tant que modalités pour obtenir un accord (une décision qui vaut pour tous) en évitant le vote à majorité. Il est possible, d'ailleurs, que dans la plupart des cas, on soit en présence d'un mélange des deux mécanismes. Si la majorité ne peut pas s'imposer ou si chaque membre a un droit de veto (et si la question n'est pas de type «oui»//«non»), toute décision collective dépend d'un compromis entre ceux qui ne sont pas d'accord sur la décision à prendre. Celui-ci suppose, en outre, que chaque décideur ait une menace crédible à faire valoir (le veto par exemple lorsqu'on est sous la contrainte de l'unanimité; mais il faut être conscient du fait que le veto n'est pas un instrument qu'on puisse faire valoir toujours et en toute occasion, ad libitum!). Le compromis peut donc se produire si les partenaires de la décision collective ont quelque chose à échanger. Mais un compromis peut aussi consister dans le fait que "a" accepte une partie de l'argument de "b".

Arrêtons-nous sur ce point. Je puis accepter l'argument d'un autre décideur car j'ai été persuadé. Ceci est d'autant plus facile si mes intérêts ne sont pas clairement définis. Ou parce que les conséquences de mon acceptation ne sont pas claires. Mais je puis décider d'accepter une partie de l'argument de l'autre si l'autre accepte une partie de mon argument. Par sens de la réalité ou de fairness, tout en n'ayant pas changé mon avis au cours de la discussion. Je suis en train de dire que l'on peut utiliser des arguments de type «public reason» pour arriver à un compromis, à une décision collective, en l'absence d'une opinion commune; mais aussi l'argument que le compromis est la seule modalité raisonnable pour arriver à une décision commune.

${ }^{25}$ Lorsqu'on parle de «vote», il faut introduire un certain nombre de précisions - sur lesquelles je ne vais pas pouvoir m'arrêter ici. D'abord, il faudrait distinguer avec Condorcet les assemblées «électives», qui permettent de choisir des chefs (le pape) ou des représentants pour des mandats à terme et renouvelables seulement par une nouvelle élection, des assemblées qu'il appelle «délibératives», celles qui prennent des décisions (celles-ci peuvent être à leur tour représentatives ou populaires). Encore plus important est de distinguer le vote à majorité simple de celui à majorité qualifiée et du vote pondéré, qu'on utilise dans les assemblées de copropriété et dans le conseil des ministres de l'Union Européenne! Jean Monnet parle de ce dernier mécanisme de prise de décision par le vote dans ses Mémoires (Paris, Fayard, 1976, 414).

${ }^{26}$ Et sans doute impossible si le nombre est très grand!

${ }^{27}$ Je dois ce renseignement à une communication personnelle du vice-président Giuliano Amato.

${ }^{28}$ Cf. P. Magnette, «La Convention européenne: argumenter et négocier dans une assemblée constituante multinationale», Revue Française de Science Politique, Vol. 54 (2004), nº 1. 
Si l'on prenait en compte les travaux de la Convention pour l'avenir de l'Europe, on verrait apparaître quelques éléments concrets liés aux considérations que j'ai essayé de développer.

Avec une précision importante. Dans le contexte de l'Union Européenne, faire valoir, même en public, les intérêts d'un sous-ensemble (un Etat-membre) n'est pas interdit en soi! Jusqu'à un certain point, le représentant d'un gouvernement peut dire: l'argument “ $x$ " n'est pas acceptable car il contredit les intérêts «légitimes $\gg{ }^{29}$ de mon pays. En fait, il se trouve qu'un gouvernement national a une légitimité démocratique (car il est élu et politiquement responsable devant le suffrage) dont était dépourvue la Convention - les membres de celle-ci n'étant ni élus ni responsables! Certes, un nombre important de conventionnels étaient mus par un intérêt de «constituants», de «pères fondateurs» (ceci n'était pas seulement évident du président Giscard et du vice-président Giuliano Amato, mais aussi d'autres, surtout des membres venants de la Commission et du Parlement Européen), mais ceci ne leur donnait pas plus de légitimité que les membres des parlements nationaux.

On peut et on doit se demander dans quelle mesure le produit final de la Convention - le nouveau traité constitutionnel - est le produit de négociations discrètes menées par Giscard avec les gouvernements des Etats-membres et dans quelle mesure il est le résultat d'échange d'arguments publics. Il est évident que la réponse est: des deux. On a pu promettre à la Pologne, en cachette (sans succès, apparemment), d'introduire dans le Préambule l'invocatio Dei qu'elle demandait avec l'Espagne, afin d'isoler celle-ci sur la question du vote pondéré dans le Conseil. Mais il est sûr que les arguments du président Giscard sur la création du Congrès de l'Union ont dû être abandonnés par celui-ci du fait qu'ils n'ont pas persuadé l'assemblée.

Intérêts et (bons) arguments sont tous les deux constamment à l'œuvre dans tout processus de décision collective. Mais la décision souveraine du vote à la majorité (qui caractérise les institutions démocratiques - directes ${ }^{30}$ ou représentatives et responsables devant le suffrage) est opposée à la décision qui se fait entre décideurs qui ne sont pas des organes démocratiques (électives et accountable) et qui ne doivent qu'échanger des arguments en vue de produire une public reason.

Philippe Urfalino fait remarquer à raison que: «L'adoption d'une règle [de prise de décision collective], son maintien, son fonctionnement, sont indissociables de considérations normatives $\rangle^{31}$. La thèse normative qui soutient la décision délibérative ou par consensus peut être reconduite à ceci: un organe - telle une Cour constitutionnelle ou la Convention dont je viens de parler - ne doit pas utiliser un mécanisme de prise de décision tel que le vote à la majorité, approprié à une assemblée populaire ou à tout organe qui discute en public et qui est politiquement responsable devant le suffrage (tel le Parlement). Dans ce dernier cas, les décideurs ainsi que leurs choix et prises de positions sont connus et ces décideurs peuvent être punis par les électeurs (hypothèse difficile à vérifier concrètement,

\footnotetext{
${ }^{29}$ Le terme introduit une nuance importante, et pas toujours facile à analyser!

30 Je pense au référendum.

${ }^{31}$ Dans l'article cité, p. 177.
} 
mais formellement vraie). On ne voit pas de quel droit des individus, compétents autant qu'on voudra, mais non élus ni responsables politiquement - tels notamment les juges d'une cour constitutionnelle ${ }^{32}$ - auraient le pouvoir de prendre à la majorité des décisions qui lient les organes élus et les citoyens. Seule une décision qui est le produit d'une délibération prenant en compte tous les points de vue peut prétendre dans ce cas à l'autorité de la chose jugée.

La Cour Constitutionnelle italienne a publié récemment une brochure dans laquelle les juges eux-mêmes décrivent leur modalité de travail. Un fragment de ce texte mérite une citation in extenso:

«The judges deliberate on how to resolve the cases before them in closed session (camera di consiglio), and in total secrecy. The Court normally meets in closed session from 9:30 a.m. to 1:00 p.m. and from 5:00 to 8:00 p.m., every other week, in conjunction with its public hearings.

It is here that the Court, under the direction of the President, debates the issues to be resolved, frames possible solutions, reaches decisions, and approves opinions. If one considers that in a year there are approximately 18 weeks of closed sessions, from Monday afternoon to Friday, and for every day of sittings the judges meet for up to 6 or 7 hours, one can calculate the amount of time that they spend together in discussion every year!

One can therefore understand the sort of longstanding rapport that grows up among the fifteen constitutional judges, in an environment the rites and rules of which are reminiscent of those of a monastery. After several months, the level of reciprocal understanding (of their respective ideas and of their ways of thinking) tends to become rather intense. Since each judge serves for nine years, one can appreciate that the experience of working in the Constitutional Court leaves a deep impression on the judges, converting the group of fifteen into something more than the sum of its parts: the Court becomes virtually a person in its own right, made up of fifteen people.

During this week of group meetings, the judges normally deal first with the cases discussed in open court, turning next to those dealt with in closed session.

Discussion may last no more than a few minutes in cases where the rapporteur proposes a solution that does not meet with objections, and is therefore immediately adopted by the Court. Or it may last entire days, depending on the complexity or controversial nature of the question at issue.

The judges work with the record and the research material before them, but it must be emphasized that the discussion is not based on a draft opinion already prepared by the rapporteur (as occurs in other Courts). Debate begins with a preliminary statement made by the rapporteur judge, which highlights any possible problems regarding the threshold admissibility of the question at issue. The report may end with a detailed proposal or with a list of various possible solutions, depending on the choice of the rapporteur.

At this point the other judges may join in the discussion, starting with the question of admissibility and then turning to the merits of the case. If the question is of relatively minor importance, it is likely that only a few judges will speak; otherwise, all will offer their thoughts. In the case of more formal discussion, the judges speak in order of age, starting with the youngest, while the President speaks last. The discussion can continue, if requested, with further observations, objections, and requests for clarification. A judge may request that the discussion be postponed until a later date, or there may be a need to acquire new material in order to examine the matter in more depth. The discussion does not necessarily follow a fixed plan. Much depends on the requests made by the judges, as

${ }^{32}$ Le cas des membres de la Convention est comparable; même s'il s'agissait en partie de membres élus des parlements nationaux et du Parlement Européen, ils n'avaient aucun mandat au moment de leur élection pour rédiger un nouveau traité, encore moins une constitution! 
well as the President's direction of the debate, although he often defers to the desires of his colleagues. The rapporteur can reply to other judges' comments, or wait until the end of the hearing to conclude the debate and offer a final proposal (which does not always coincide with the proposal he presented at the beginning). It is here, above all, that one can measure the efficacy and utility of the group discussion, which can generate objections to the case as presented by the rapporteur as well as suggestions for different grounds on which to base the decision.

One must consider that the final decision of the Court consists not only of the formal judgment itself (such as a declaration of unconstitutionality, a declaration that the certified question is unfounded, or declarations that the question itself is inadmissible), but also, and sometimes above all, of the grounds for the decision spelled out in the opinion of the Court. There may be agreement on the ultimate result, but dissent with respect to the grounds for that result. The latter are important primarily because they constitute more than the judgment itself - the nucleus of the precedents referred to in cases that the Court is called to decide in the same or similar matters in the future; and also because a single judgment might be supported by reasoning that produces different effects. For example, a decision that rejects a constitutional challenge on the grounds that the impugned provision is constitutional is very different from one that declares the same question unfounded because the challenged law should be interpreted in a different way from that indicated by the judge. Therefore, settling on the grounds for a decision is sometimes more important than deciding whether or not the law is unconstitutional. This may account for the determined and protracted nature of some discussions in closed session.

Majority Decisions?

Like any other group of thinking heads, the Constitutional Court can find itself divided. With fifteen judges, some dissent is likely, despite the fact that all the judges rely on the same Constitution and that their long hours of collaboration favor the formation of common views. Accordingly, the Court, like other collegial bodies, must arrive at decisions by majority vote. A formal vote is held only when there is a lack of unanimity (for example, in support of the rapporteurs proposed resolution) or even a clear majority of similar viewpoints, or if a judge requests such a vote. The President calls the vote, thus bringing deliberations to a close.

The practices of the Court may vary depending on the styles and attitudes of the President and the other judges, but the basic goal is to achieve the broadest possible consensus among the judges. For this reason, discussions are sometimes extended to look for compromise solutions, or at least solutions that avoid sharp divisions within the Court. The compromise can often consist of a decision that does not resolve the question definitively, for example by declaring a certified question inadmissible rather than rejecting it on the merits. Less dramatically, the Court might simply narrow the sweep of the reasoning in its opinion. This practice is probably driven in part by the current lack of a vehicle, such as dissenting opinions, which are published in Germany and Spain, for judges to register their disagreement with the majority view.

The general practice of the Court is to accept or reject the rapporteurs final proposal. Sometimes, if a preliminary question emerges (e.g., regarding the admissibility of the certified question) the Court first takes a vote on the rapporteurs proposal regarding this issue and then, if necessary, on the rapporteurs proposal on the merits of the case. If the rapporteur proposes a series of options, ranking them in order of preference, the Court considers these proposals in the order suggested by the rapporteur. This agenda-setting power is perhaps the most significant power in the hands of the rapporteur, whose personality can at times contribute to the formation of a majority in support of his proposal. All judges present during the deliberations must vote for or against any proposal put to the vote; they may not abstain. Furthermore, all the judges present at the beginning of the discussion on a case, either at the public hearing or in closed session, must take part in deliberations until the end and cannot, as is often the case in political assemblies, «leave 


\begin{abstract}
the room» to effectively abstain from voting. Finally, the composition of the Court cannot change during the discussion of a case.

If the Court is made up of an even number of judges and the vote is evenly split, then the outcome is determined by the vote of the President (or whoever presides over the sitting). This is the only occasion when the President exercises any power greater than that of the other judges. In all other circumstances, his vote is worth the same as that of the others. His influence naturally derives from his authority vis-à-vis his colleagues, but there are no internal hierarchies within the Court, only varying personalities and opinions ${ }^{33}$.
\end{abstract}

Comme on le voit en lisant ce texte, le vote n'est pas exclu de la décision par délibération, mais il ne représente ni le mécanisme essentiel, ni celui considéré souhaitable par la Cour. Dans un texte récent de l'ancien président de la Cour Constitutionnelle italienne, Gustavo Zagrebelsky revient sur la question de la règle de décision de l'organe de contrôle de constitutionnalité:

«Je ne dévoile pas un secret en disant que, sur les questions les plus importantes, celle qui concernent la substance du droit constitutionnel, on essaye de ne pas voter ou, plus exactement, on fait l'effort de décider sans qu'il soit nécessaire de recourir au vote ou en réduisant celui-ci à une simple formalité [...] l'optimum serait l'unanimité. L'objectif réaliste: la solution la plus partagée» ${ }^{34}$.

Institut Jean Nicod, CNRS, Paris et New York University

33 The Italian Constitutional Court, Corte Costituzionale - Roma, June 2004, pp. 45-48.

${ }^{34}$ Discours tenu à l'occasion de l'attribution du prix Giuseppe Chiarelli, Corte costituzionale Palazzo della Consulta, Roma, 20 ottobre 2004 [ce texte n'est pas encore publié, je remercie l'auteur de me l'avoir communiqué]. 